\title{
Studies of space-charge effects in ultrashort electron bunches
}

\author{
Gwenaël Fubiani, ${ }^{1}$ Wim Leemans and Eric Esarey \\ Center for Beam Physics, Ernest Orlando Lawrence Berkeley National Laboratory, University of \\ California, Berkeley CA 94720
}

\begin{abstract}
Laser-driven plasma-based accelerators are capable of producing ultrashort electron bunches in which the longitudinal size is much smaller than the transverse size. We present theoretical studies of the transport of such electron bunches in vacuum. Space charge forces acting on the bunch are calculated using an ellipsoidal bunch shape model. The effects of space charge forces and energy spread on longitudinal and transverse bunch properties are evaluated for various bunch lengths, energies and amount of charge.
\end{abstract}

\section{INTRODUCTION}

Plasma-based accelerators offer the possibility of providing compact, high energy electron accelerators [1]. Plasmas can sustain ultrahigh electric fields, thus providing for rapid acceleration. In addition, plasma-based accelerators can generate ultrashort electron bunches with a large amount of electrons per bunch. In a plasma, the wavelength of the accelerating field is the plasma wavelength, $\lambda_{p}=2 \pi c / \omega_{p}$, where $\omega_{p}=\left(4 \pi n_{e} e^{2} / m_{0}\right)^{1 / 2}$ is the plasma frequency and $n_{e}$ is the electron plasma density. In engineering units, the plasma wavelength is

$$
\lambda_{p}[\mu \mathrm{m}] \simeq 330\left(n_{e}\left[\mathrm{~cm}^{-3}\right]\right)^{-1 / 2} .
$$

For example, a laser wakefield accelerator (LWFA) in the standard regime typically has a density on the order of $n_{e} \simeq 10^{18} \mathrm{~cm}^{-3}$ and a plasma wavelength on the order of $\lambda_{p} \simeq 30 \mu \mathrm{m}$ (100 fs). If a mono-energetic electron bunch is injected into a wakefield such that it is accelerated while maintaining a small energy spread, then it is necessary that the bunch occupy a small fraction of the wake period, i.e., the bunch must be ultrashort, on the order of a few femtoseconds. Test particle simulations of the colliding pulse LWFA injector [2] - [4], in which two counterpropagating laser pulses are used to inject electrons from the background plasma directly into the wake, indicate the production of a trapped bunch $\left(\sim 10^{7}\right.$ electrons $)$ with a low

1) Also at University of Paris XI, France 
energy spread $(<1 \%)$, low normalized emittance $(\sim 1 \mathrm{~mm}-\mathrm{mrad})$, and of ultrashort duration ( $\sim 1 \mathrm{fs}$ ) may be possible. Such test particle simulations, however, neglected the space charge effects of the accelerated bunch.

Space charge effects can limit the amount of charge that can be transported in an ultrashort, tightly focused electron bunch, i.e., space charge can lead to a increase in both the longitudinal and transverse bunch dimensions. In a LWFA, space charge effects may not be of concern while the bunch is in the plasma wave, since the longitudinal and transverse fields of the wake are typically much greater than the space charge forces of the bunch. This is not the case, however, as the bunch exits the plasma into a vacuum region with no applied fields. In this case, space charge can lead to a rapid blow-up of the bunch. This paper will model the dynamics of ultrashort bunches in vacuum under the assumptions that (i) the spatial extent of the bunch remains ellipsoidal and (ii) the charge density within the bunch remains uniform.

\section{INFINITE CYLINDRICAL BEAM}

When a beam has a longitudinal size much larger than the transverse size, one can assume that the space charge force that occurs on the beam is only transverse. Since the longitudinal field is almost zero in this case, one can easily apply Gauss's law to calculate the transverse electric field. The radial electric field $\mathrm{E}_{r^{\prime}}$ in the beam frame (denoted by a prime) is given by

$$
\mathrm{E}_{r^{\prime}}=\rho^{\prime} r^{\prime} /\left(2 \epsilon_{0}\right)
$$

where $\rho^{\prime}$ the charge density in the beam frame (a frame moving with the average velocity of the beam electrons). The quantities in the lab frame are related to those in the beam frame by the Lorentz transforms [5],

$$
\begin{aligned}
& \mathbf{E}=\gamma\left(\mathbf{E}^{\prime}-\beta \times \mathbf{B}^{\prime}\right)-\gamma^{2}(\gamma+1)^{-1} \beta\left(\beta \cdot \mathbf{E}^{\prime}\right) \\
& \mathbf{B}=\gamma\left(\mathbf{B}^{\prime}+\beta \times \mathbf{E}^{\prime}\right)-\gamma^{2}(\gamma+1)^{-1} \beta\left(\beta \cdot \mathbf{B}^{\prime}\right)
\end{aligned}
$$

along with $\mathrm{E}_{r}=\gamma \mathrm{E}_{r^{\prime}}^{\prime}, \mathrm{B}_{\theta}=\gamma \beta \mathrm{E}_{r^{\prime}}^{\prime}, r^{\prime}=r, z^{\prime}=\gamma z$, and $\rho^{\prime}=\rho / \gamma$, where $\gamma=$ $\left(1-\beta^{2}\right)^{-1 / 2}$ is the relativistic factor, $\beta=v / c$ and $v$ is the beam velocity (assumed to be along the $z$-axis). Thus, the radial Lorentz-Coulomb force in the lab frame is

$$
\mathrm{F}_{r}^{\text {self }}=q\left(\mathrm{E}_{r}+\beta \times \mathrm{B}_{\theta}\right)=q \gamma\left(1-\beta^{2}\right) \mathrm{E}_{r^{\prime}}^{\prime}=\rho r /\left(2 \gamma^{2} \epsilon_{0}\right) .
$$

Newton's equation of motion can be easily computed under the assumptions $\beta \sim 1, \gamma \sim \gamma_{0}$ and $v_{\theta}(t=0)=0$ (laminar flux) [6], i.e.,

$$
\ddot{r}+\dot{\gamma} \dot{r} / \gamma=\mathrm{F}_{r}^{\text {self }} /\left(\gamma m_{0}\right) .
$$

where the dot denotes $d / d t$. Furthermore, assuming that the change in energy $\dot{\gamma} / \gamma$ remains small, and letting $s=c t$, we can approximate 




FIGURE 1. Transverse motion of a single electron with zero initial divergence. The motion is only due to space charge forces in the infinite beam limit.

$$
\gamma m_{0} d^{2} r / d s^{2} \simeq \mathrm{F}_{r}^{s e l f} / c^{2}
$$

Figure 1 shows the results of numerically solving Eq. (4), assuming a relatively long electron bunch with an average energy of $E_{0}=20 \mathrm{MeV}$, a bunch radius of $\Delta r_{0}=4 \mu \mathrm{m}$, a bunch length of $\Delta z_{0}=0.5 \mu \mathrm{m}$ (the theory assumed an infinite beam, but it is necessary to define a bunch length for the calculation of an equivalent charge density), an angular spread of $\theta_{0}=0 \mathrm{mrad}$, a total charge of $Q=1 p C$ and a charge density $\rho=Q /\left(\pi \Delta r_{0}^{2} \Delta z_{0}\right)$. Figure 1 is useful in that it shows the effects of space charge that one expects in the limit of a very long beam.

It is insightful to compare these results to those that are obtained from a more precise calculation that takes into consideration the finite duration of the bunch. The next section will discuss the space charge model for an ultrashort bunch, under the assumption of an ellipsoidal bunch shape. In this particular case one can calculate the potential $U$ and the electric field everywhere at the surface and inside the ellipsoid. We will compare these two models and define the range where the simple case of an infinite beam can still adequately describe the bunch evolution.

\section{TRANSVERSE AND LONGITUDINAL SPACE-CHARGE FORCES}

The equations of motion that we use to model the effects of space charge on the transverse and longitudinal motion of a single beam electron are given by

$$
\frac{d^{2} r}{d s^{2}}=\frac{1}{\gamma_{0} m_{0} v_{0}^{2}} F_{r}^{s e l f}
$$




$$
\frac{d^{2} \sigma}{d s^{2}}=\frac{1}{\gamma_{0}^{3} m_{0} v_{0}^{2}} F_{s}^{s e l f}
$$

where $F_{r}^{\text {self }}$ and $F_{s}^{\text {self }}$ are the effective space charge forces in the transverse and longitudinal directions, respectively, $s=\int d t v_{s}+s_{0}$ is the longitudinal position of the electron with velocity $v_{s}, s_{0}$ is a constant, $\sigma=s-v_{0} t_{s}(s)$ is the longitudinal distance between the electron and that of the synchronous particle located at the bunch center $\Delta s=v_{0} t_{s}(s), v_{0}$ is the average velocity of the bunch (assumed to be constant) and $t_{s}(s)=\int d s / v_{s}$. Here it is assumed that the electron is highly relativistic, $\beta \simeq 1$, with an energy near the average energy of the bunch, $\gamma \simeq \gamma_{0}$. Next we outline the derivations of Eqs. (5) and (6).

\section{Transverse motion}

Equation (3) describes in cylindrical coordinates the motion of a single electron of energy $\gamma m_{0} c^{2}$ and charge $q=-e$. Assuming $v_{\theta}=0$ (laminar beam), $\dot{\gamma} / \gamma$ small $\left(\gamma \simeq \gamma_{0}\right.$, i.e., the electron energy is near the average energy of the bunch) and $\gamma_{\perp} \ll \gamma_{\|} \simeq \gamma_{0}$, the transverse equation of motion is given by

$$
\ddot{r}=\frac{\mathrm{F}_{r}^{\text {self }}}{\gamma_{0} m_{0}} .
$$

Furthermore, for the transverse motion, it is adequate to approximate $s \simeq v_{0} t+s_{0}$. Hence,

$$
\frac{d^{2} r}{d s^{2}}=\frac{\mathrm{F}_{r}^{s e l f}}{\gamma_{0} m_{0} v_{0}^{2}}
$$

\section{Longitudinal motion}

We define $\Delta E_{s}$ as the energy spread induced by the longitudinal space charge force, i.e.,

$$
\Delta E_{s}=\int d s \mathrm{~F}_{s}^{s e l f}
$$

The energy spread of the electrons in the bunch is assumed small compared to the average energy $E_{0}=\gamma_{0} m_{e} c^{2}\left(\gamma_{0} \gg 1\right)$ of the bunch, i.e., $\left|\Delta E_{s}\right| / E_{0} \ll 1$. The variable $\eta=\Delta E_{s} / E_{0}$ is then appropriate to describe the longitudinal dynamics of the bunch [7], [8].

It is then straightforward to show that the spread in longitudinal momentum $\Delta p_{s}$ due to the longitudinal space charge force is related to the variable $\eta$ by

$$
\frac{\Delta p_{s}}{p_{0}}=\frac{1}{\beta_{0}} \sqrt{(1+\eta)^{2}-\frac{m_{0} c^{2}}{E_{0}^{2}}}-1=f(\eta),
$$


where $\beta_{0}=v_{0} / c$ and $p_{0}=\gamma_{0} \beta_{0} m_{0} c$. Note that the derivatives of $f(\eta)$ with respect to $\eta$ are given by

$$
\frac{d f(\eta)}{d \eta}=\frac{1}{\beta_{0} \beta}, \frac{d f(0)}{d \eta}=\frac{1}{\beta_{0}^{2}}, \frac{d^{2} f(0)}{d \eta^{2}}=\frac{1}{\beta_{0}^{4} \gamma_{0}^{2}} .
$$

We now define the longitudinal position variable $\sigma=s-v_{0} t_{s}(s)$ and find its time evolution by expanding $f(\eta)=\Delta p_{s} / p_{0}$ about $\eta=0$. Note that

$$
\frac{d \sigma}{d s}=1-v_{0} \frac{d t_{s}}{d s}=1-\frac{v_{0}}{v_{s}}=1-\beta_{0}^{2} \frac{d f(\eta)}{d \eta} .
$$

If $\eta \ll 1$, then $d f(\eta) / d \eta \simeq d f(0) / d \eta+\eta d^{2} f(0) / d \eta^{2}$. This implies $d \sigma / d s \simeq \beta_{0}^{-2} \gamma_{0}^{-2} \eta$ and, hence,

$$
\frac{d^{2} \sigma}{d s^{2}}=\frac{1}{\gamma_{0}^{3} m_{0} v_{0}^{2}} \mathrm{~F}_{s}^{s e l f},
$$

which is valid for relativistic bunches, $\gamma_{0} \gg 1$, with small energy spreads $|\eta|=$ $\left|\gamma-\gamma_{0}\right| / \gamma_{0} \ll 1$.

\section{ELLIPSOIDAL BEAM THEORY}

We next calculate the electrostatic potential of the bunch in $3 \mathrm{D}$ by making a summation over all the charges inside the bunch. The model described below closely follows references [7]- [9]. The 3D bunch is described as an ellipsoid that maintains its ellipsoidal shape as it evolves. In the lab frame, the longitudinal bunch size is defined as $\sigma_{s}$ along the longitudinal coordinate $\sigma$, and the transverse bunch sizes as $\sigma_{x}$ and $\sigma_{y}$ along the transverse coordinates $x$ and $y$. In the frame moving with the bunch, the bunch boundary is assumed to be an ellipse given by

$$
\frac{x^{\prime 2}}{\sigma_{x}^{2}}+\frac{y^{\prime 2}}{\sigma_{y}^{2}}+\frac{\sigma^{\prime 2}}{\gamma_{0}^{2} \sigma_{s}^{2}}=1 .
$$

Furthermore, within this ellipse, the charge density of the bunch is assumed to be uniform.

In the interior of the ellipse, the electrostatic potential in the bunch frame (denoted by a prime) is a quadratic function of $x^{\prime}, y^{\prime}$ and $\sigma^{\prime}$,

$$
U^{\prime}=-A x^{2}-B y^{\prime 2}-C{\sigma^{\prime}}^{2}+D
$$

where $A, B, C$, and $D$ are constants. The electric field in the bunch frame is $\mathbf{E}^{\prime}=-\nabla \mathbf{U}^{\prime}$. Integrating over the ellipsoid volume, and using the expression of the total charge $Q=(4 \pi / 3) \sigma_{x} \sigma_{y} \sigma_{s} \rho$, gives

$$
E_{x}^{\prime}=\frac{3}{8 \pi \epsilon_{0}} Q I_{1} x^{\prime} \quad\left(A=\frac{3}{16 \pi \epsilon_{0}} Q I_{1}\right),
$$




$$
\begin{aligned}
& E_{y}^{\prime}=\frac{3}{8 \pi \epsilon_{0}} Q I_{2} y^{\prime} \quad\left(B=\frac{3}{16 \pi \epsilon_{0}} Q I_{2}\right), \\
& E_{s}^{\prime}=\frac{3}{8 \pi \epsilon_{0}} Q I_{3} \sigma^{\prime} \quad\left(C=\frac{3}{16 \pi \epsilon_{0}} Q I_{3}\right),
\end{aligned}
$$

where $I_{1}, I_{2}$ and $I_{3}$ are the elliptical integrals given by

$$
\begin{gathered}
I_{1}=\int_{0}^{\infty} \frac{d \tau}{\left(\sigma_{x}^{2}+\tau\right) \sqrt{\phi(\tau)}}, \\
I_{2}=\int_{0}^{\infty} \frac{d \tau}{\left(\sigma_{y}^{2}+\tau\right) \sqrt{\phi(\tau)}}, \\
I_{3}=\int_{0}^{\infty} \frac{d \tau}{\left(\gamma_{0}^{2} \sigma_{s}^{2}+\tau\right) \sqrt{\phi(\tau)}}
\end{gathered}
$$

with $\phi(\tau)=\left(\sigma_{x}^{2}+\tau\right)\left(\sigma_{y}^{2}+\tau\right)\left(\gamma_{0}^{2} \sigma_{s}^{2}+\tau\right)$.

The fields in the lab frame are given by Eq. (2), along with $E_{x}=\gamma_{0} E_{x}^{\prime}, E_{y}=$ $\gamma_{0} E_{y}^{\prime}, E_{s}=E_{s}^{\prime}, B_{x}=\beta_{0} \gamma_{0} E_{y}^{\prime}, B_{y}=-\beta_{0} \gamma_{0} E_{x}^{\prime}, B_{s}=0$ (we assumed $\mathbf{B}^{\prime} \sim \mathbf{0}$, i.e., the relative motion between the electrons inside the bunch is almost zero), $r^{\prime}=r$, $y^{\prime}=y$ and $\sigma^{\prime}=\gamma_{0} \sigma$.

We extract the Lorentz-Coulomb force $\mathbf{F}^{\text {self }}=q(\mathbf{E}+\mathbf{v} \times \mathbf{B} / c)$ from the fields,

$$
\begin{gathered}
F_{x}=\frac{3}{8 \pi \epsilon_{0}} \frac{e Q}{\gamma_{0}} x \int_{0}^{\infty} \frac{d \tau}{\left(\sigma_{x}^{2}+\tau\right) \sqrt{\left(\sigma_{x}^{2}+\tau\right)\left(\sigma_{z}^{2}+\tau\right)\left(\gamma_{0}^{2} \sigma_{s}^{2}+\tau\right)}} \\
F_{y}=\frac{3}{8 \pi \epsilon_{0}} \frac{e Q}{\gamma_{0}} y \int_{0}^{\infty} \frac{d \tau}{\left(\sigma_{z}^{2}+\tau\right) \sqrt{\left(\sigma_{x}^{2}+\tau\right)\left(\sigma_{z}^{2}+\tau\right)\left(\gamma_{0}^{2} \sigma_{s}^{2}+\tau\right)}} \\
F_{s}=\frac{3}{8 \pi \epsilon_{0}} e Q \gamma_{0} \sigma \int_{0}^{\infty} \frac{d \tau}{\left(\gamma_{0}^{2} \sigma_{s}^{2}+\tau\right) \sqrt{\left(\sigma_{x}^{2}+\tau\right)\left(\sigma_{z}^{2}+\tau\right)\left(\gamma_{0}^{2} \sigma_{s}^{2}+\tau\right)}}
\end{gathered}
$$

Assuming that the beam has a cylindrical symmetry, i.e., $\sigma_{r} \equiv \sigma_{x}=\sigma_{y}$, the forces are then given by,

where

$$
F_{r}=\frac{3}{8 \pi \epsilon_{0}} \frac{e Q}{\gamma_{0}} r I_{r}, \quad F_{s}=\frac{3}{8 \pi \epsilon_{0}} e Q \gamma_{0} \sigma I_{s}
$$

$$
\begin{aligned}
& I_{r}=\int_{0}^{\infty} d \tau(a+\tau)^{-2}(b+\tau)^{-1 / 2}, \\
& I_{s}=\int_{0}^{\infty} d \tau(a+\tau)^{-1}(b+\tau)^{-3 / 2},
\end{aligned}
$$

with $a=\sigma_{r}^{2}$ and $b=\gamma_{0}^{2} \sigma_{s}^{2}$. The integrals $I_{r}$ and $I_{s}$ can be calculated analytically,

$$
I_{r}= \begin{cases}\frac{\pi}{2(a-b)^{3 / 2}}-\frac{b^{1 / 2}}{a(a-b)}-(a-b)^{-3 / 2} \tan ^{-1}\left[b^{1 / 2}(a-b)^{-1 / 2}\right], & \text { for } a>b, \\ \frac{b^{1 / 2}}{a(b-a)}+\frac{1}{2}(b-a)^{-3 / 2} \ln [(\sqrt{b}-\sqrt{b-a}) /(\sqrt{b}+\sqrt{b-a})], & \text { for } a<b,\end{cases}
$$


$I_{s}= \begin{cases}-\frac{\pi}{(a-b)^{3 / 2}}+\frac{2}{b^{1 / 2}(a-b)}+2(a-b)^{-3 / 2} \tan ^{-1}\left[b^{1 / 2}(a-b)^{-1 / 2}\right], & \text { for } a>b \\ -\frac{2}{b^{1 / 2}(b-a)}-(b-a)^{-3 / 2} \ln [(\sqrt{b}-\sqrt{b-a}) /(\sqrt{b}+\sqrt{b-a})], & \text { for } a<b\end{cases}$

and $I_{r}=I_{s}=(2 / 3) a^{-3 / 2}$ for $a=b$.

In the limit $a \ll b$ (i.e $\sigma_{r} \ll \gamma_{0} \sigma_{s}$ ), the above expressions can be expanded to yield

$$
\begin{gathered}
I_{r}=\frac{1}{b^{1 / 2} a}+b^{-3 / 2}[1+(1 / 2) \ln (4 b / a)][1+O(a / b)], \\
I_{s}=b^{-3 / 2}[2+\ln (4 b / a)][1+O(a / b)] .
\end{gathered}
$$

Notice that $I_{s} / I_{r} \sim O(a / b)$. Hence, to leading order in $a / b, I_{r} \sim 1 /\left(\gamma_{0} \sigma_{s} \sigma_{r}^{2}\right)$ and $I_{s} \sim 0$ and we recover the forces on an infinite and cylindrical beam, i.e., $F_{r}=e \rho r /\left(2 \gamma_{0}^{2} \epsilon_{0}\right)$ and $F_{s} \sim 0$, where the volume of the ellipsoid is $V_{\text {ell }}=3 /\left(4 \pi \sigma_{r}^{2} \sigma_{s}\right)$.

\section{ENVELOPE EQUATIONS}

\section{Space charge dominated}

The trajectory of any particle within the bunch can be solved if the bunch radius and length (boundaries of the ellipse), $\sigma_{r}$ and $\sigma_{s}$, are know as a function of propagation distance. Now under the condition of a space charge dominated beam, the forces on a particle scale as $F_{r} \sim r$ and $F_{s} \sim s$. Specifically, a particle at a larger radius will remain at a larger radius compaered to a particle at a smaller radius. We will estimate the evolution of the beam envelope by replacing $r$ with $\sigma_{r}$ and $\sigma$ with $\sigma_{s}$ in Eqs. (5) and (6), i.e.,

$$
\begin{gathered}
\sigma_{r}^{\prime \prime}=\frac{K_{r}\left(\sigma_{r}, \sigma_{s}\right)}{\sigma_{r}}, \quad \sigma_{s}^{\prime \prime}=\frac{K_{s}\left(\sigma_{r}, \sigma_{s}\right)}{\sigma_{s}}, \\
K_{r}=\frac{3}{8 \pi \epsilon_{0}} \frac{e Q}{\gamma_{0}} I_{r}\left(\sigma_{r}, \sigma_{s}\right), \quad K_{s}=\frac{3}{8 \pi \epsilon_{0}} e Q \gamma_{0} I_{s}\left(\sigma_{r}, \sigma_{s}\right),
\end{gathered}
$$

where the contribution of finite emittance to the envelope equations has been neglected.

\section{Emittance dominated}

It is interesting to compare the space charge dominated regime to the emittance dominated regime. In free space, neglecting space charge forces, the envelope equations for the electron bunch length and radius are given by

$$
\sigma_{r}^{\prime \prime}-\frac{\epsilon_{r}^{2}}{\sigma_{r}^{3}}=0, \quad \sigma_{s}^{\prime \prime}-\frac{\epsilon_{s}^{2}}{\sigma_{s}^{3}}=0,
$$


where $\epsilon_{r}$ and $\epsilon_{s}$ are the rms unnormalized emittances in the transverse and longitudinal direction, which are given by $[10]$

$$
\epsilon_{s}=\left(\sigma_{s}^{0} / \gamma_{0}^{2}\right) \Delta p_{s} / p_{0}, \quad \epsilon_{r}=\left(\left\langle r^{2}\right\rangle\left\langle r^{\prime 2}\right\rangle-\left\langle r r^{\prime}\right\rangle^{2}\right)^{1 / 2}
$$

where $\sigma_{s}^{0}$ is the initial bunch length, $\Delta p_{s}(\eta) / p_{0}$ is the longitudinal momentum spread, and the angular brackets denote an averaging over the particle distribution. The usual definitions for the normalized emittances are given by

$$
\epsilon_{r, n}=\gamma_{0} \beta_{0} \epsilon_{r}, \quad \epsilon_{s, n}=\gamma_{0}^{3} \beta_{0} \epsilon_{s}
$$

Equation (9) can be readily integrated to yield

$$
\begin{aligned}
& \sigma_{r}^{2}(s)=\left(\sigma_{r}^{0}\right)^{2}+2 \sigma_{r}^{0} \sigma_{r}^{\prime 0} s+\left[\left(\epsilon_{r} / \sigma_{r}^{0}\right)^{2}+\left(\sigma_{r}^{\prime 0}\right)^{2}\right] s^{2}, \\
& \sigma_{s}^{2}(s)=\left(\sigma_{s}^{0}\right)^{2}+2 \sigma_{s}^{0} \sigma_{s}^{\prime 0} s+\left[\left(\epsilon_{s} / \sigma_{s}^{0}\right)^{2}+\left(\sigma_{s}^{\prime 0}\right)^{2}\right] s^{2},
\end{aligned}
$$

where the initial conditions $\sigma(0)=\sigma^{0}$ and $\sigma^{\prime}(0)=\sigma^{\prime 0}$ have been assumed.

\section{NUMERICAL SOLUTIONS AND COMPARISONS}

\section{Studies of ultrashort bunches}

Next we will consider two cases: (i) electron bunches with low $\Delta E / E_{0}$ such as we expect to produce with colliding pulse LWFA injectors [2] - [4], and (ii) electron beams with $100 \% \Delta E / E_{0}$ such as produced with self-modulated laser wakefield accelerators (SM-LWFA) [11] - [15].

\section{Beam envelope growth in the colliding pulse LWFA regime}

Typical bunch characteristics expected from a colliding pulse injector [2] - [4] are a total charge $Q \sim 1 p C$, a low energy spread $(\eta \sim 1 \%)$, a transverse size of the order of the laser spot size $\left(\sigma_{r} \sim 5 \mu \mathrm{m}\right)$ and an ultrashort bunch duration $(\Delta \tau \sim 1$ fs, i.e., $\left.\sigma_{s} \sim 0.1-1 \mu m\right)$. Figures 2,4 and 3 plot the bunch radius $\sigma_{r}(s)$ and length $\sigma_{s}(s)$ for the space charge dominated regime, Eq. (8), as a function of propagation distance and charge (at $s=50 \mathrm{~mm}$ ) assuming several electron bunch energies $E_{0}=20-40 \mathrm{MeV}$, an initial bunch radius $\sigma_{r}^{0}=4 \mu \mathrm{m}$, an initial bunch length $\sigma_{s}^{0}=0.5 \mu \mathrm{m}$, an initial divergence $\sigma_{r}^{\prime 0}=\sigma_{s}^{\prime 0}=0$ (initially at the beam focus), and a total amount of charge from 1 to $100 p C$. For comparison, we also plot results from the emittance dominated regime, Eq. (9), for a normalized transverse emittance of $\epsilon_{r, n}=0.1,0.5$ and $1.0 \mathrm{~mm}-\mathrm{mrad}$, and a normalized longitudinal emittance of $\epsilon_{s, n}=6 \times 10^{-5} \mathrm{~mm}-\mathrm{mrad}$ and $\epsilon_{s, n}=6 \times 10^{-4} \mathrm{~mm}-\mathrm{mrad}$, which corresponds to an energy spread $\eta$ of $1 \%$ and $10 \%$. These figures clearly show that a fairly high energy electron bunch $(\sim 20 \mathrm{MeV})$ with a total amount of charge of several $p C$ 




FIGURE 2. Transverse beam size versus propagation distance with space charge $(Q=1 \mathrm{pc})$ for a (a) 20 and (b) $40 \mathrm{MeV}$ bunch. Curves (c) and (d) are for no space charge and normalized emittances of $0.1 \mathrm{~mm}-\mathrm{mrad}$

produced by colliding pulse injection can rapidly blow-up via space charge due to its very compact size if $\epsilon_{r, n} \sim 0.1 \mathrm{~mm}$-mrad. Below $30 p C$ per bunch the beam is emittance dominated when its value is of the order of $\epsilon_{r, n}=1 \mathrm{~mm}-\mathrm{mrad}$, which is a typical value obtained from numerical simulations, i.e., in this case one can neglect the effect of space charge. Above this value space charge must be considered and clearly participates in the beam growth. Around $50 \mathrm{pC}$ both phenomenon must be

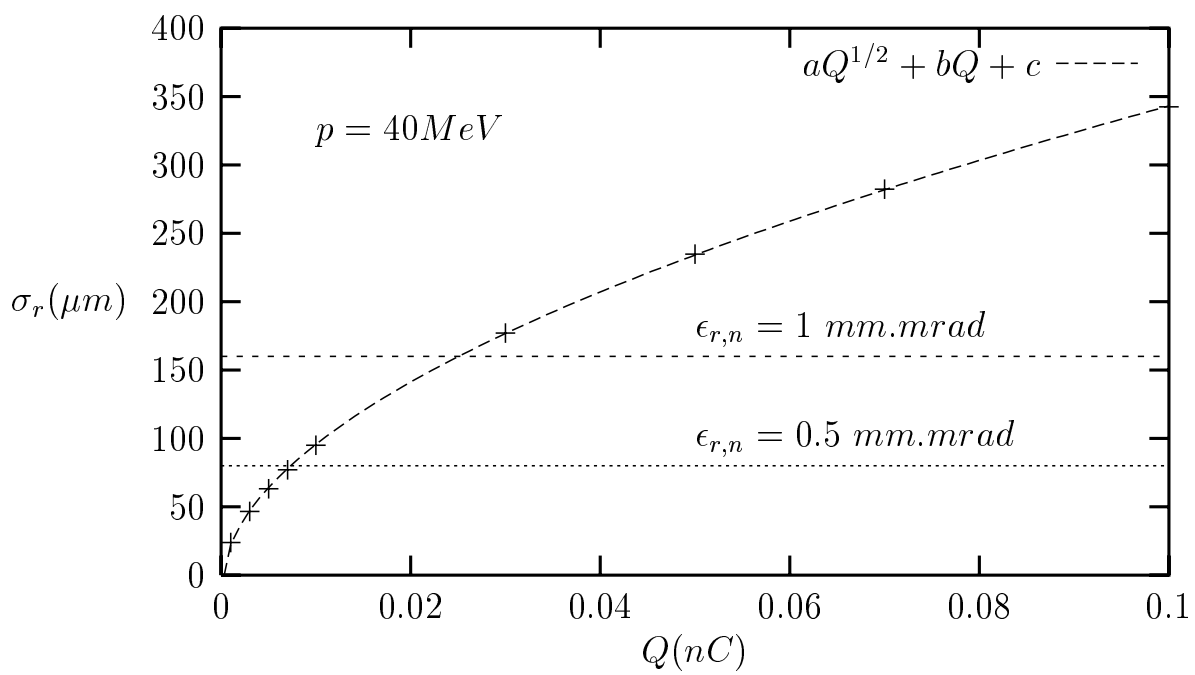

FIGURE 3. Transverse beam size (at $s=50 \mathrm{~mm}$ ) versus charge with space charge for a 40 $\mathrm{MeV}$ bunch. The horizontal lines are for no space charge and normalized emittance of 0.5 and 1 mm-mrad. 


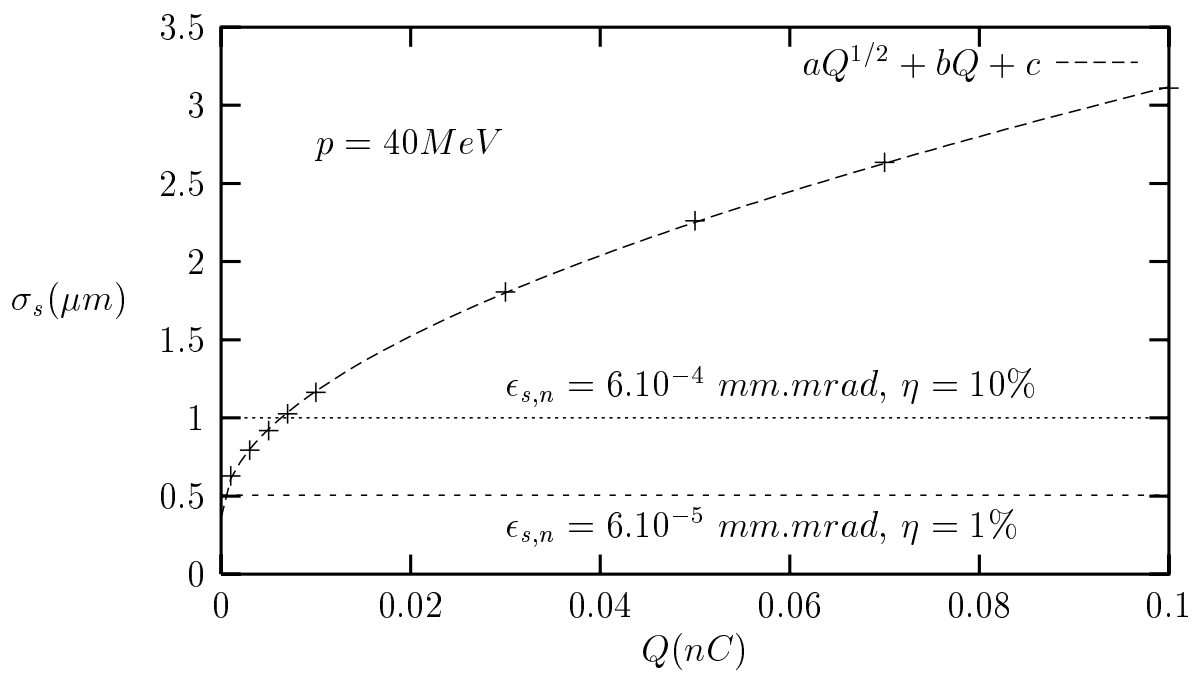

FIGURE 4. Longitudinal beam size (at $s=50 \mathrm{~mm}$ ) versus charge with space charge for a 40 $\mathrm{MeV}$ bunch. The horizontal lines are for no space charge and normalized longitudinal emittance of $6 \times 10^{-5}$ and $6 \times 10^{-4} \mathrm{~mm}-\mathrm{mrad}$.

simultaneously studied and Eqs. (8) and (9) cannot be decoupled for the transverse motion, i.e., the coupled envelope equation is,

$$
\sigma_{r}^{\prime \prime}=\frac{K_{r}\left(\sigma_{r}, \sigma_{s}\right)}{\sigma_{r}}+\frac{\epsilon_{r}^{2}\left(\sigma_{r}, \sigma_{s}\right)}{\sigma_{r}^{3}} .
$$

Conversely, one can consider that above a charge of $50 p C$ per bunch the beam is space charge dominated.

On the other hand from Fig. 4, one can see that the longitudinal motion for a $5 p C$ bunch is already in a space charge dominated regime if we assume an initial energy spread of $\eta=1 \%$. The longitudinal normalized emittance in this case is $\epsilon_{s, n}=6 \times 10^{-5} \mathrm{~mm}-\mathrm{mrad}$.

\section{Beam envelope growth in the SM-LWFA regime}

In the SM-LWFA regime, the electron energy distribution is assumed to be similar to that observed experimentally [11] - [15], as shown in Fig.5, with a total charge per bunch on the order of $1 \mathrm{nC}$. To simulate the bunch size evolution, the method we use is to take the number of electrons, $N_{i}$, present in a small energy bin about the energy $E_{i}$, and to calculate the beam sizes $\sigma_{r i, s i}$ for this group of electrons using Eq. (8) under the influence of the total bunch charge.

The overall bunch sizes $\sigma_{r, s}$ are estimated by summing the contribution of each group of electrons to the overall bunch profile, under the assumption that the 


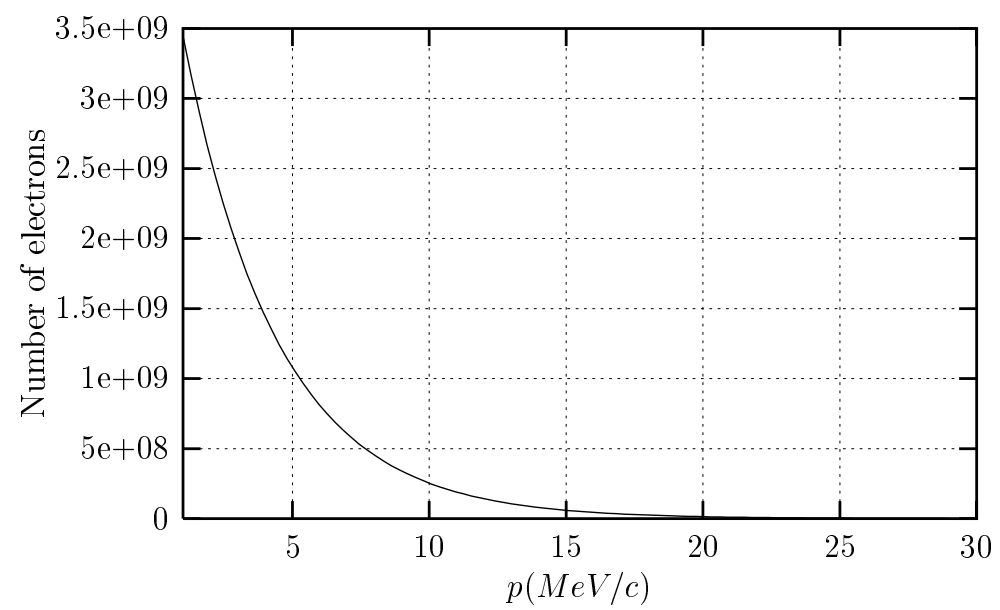

FIGURE 5. Momentum distribution model for an electron bunch produced by a SM-LWFA.

profiles for each group, as well as the overall profile, is flattop, i.e.,

$$
\left\langle\sigma_{r}^{2}\right\rangle=\sum_{i=0}^{n} N_{i} \sigma_{r i}^{2} / \sum_{i=0}^{n} N_{i}, \quad\left\langle\sigma_{s}^{2}\right\rangle=\sum_{i=0}^{n} N_{i} \sigma_{s i}^{2} / \sum_{i=0}^{n} N_{i}
$$

Using this model, it is found that the beam spot size blows-up rapidly due to the large amount of low energy electrons (energy spread per bunch $\eta$ is close to $100 \%$ ), which are more sensitive to space charge forces. Figure 6 shows the beam radius versus charge after $s=50 \mathrm{~mm}$ from the numerical solution of Eqs. (8) and (10), assuming $\sigma_{r}^{0}=5 \mu \mathrm{m}, \sigma_{s}^{0}=10 \mu \mathrm{m}, \sigma_{s}^{\prime 0}=\sigma_{r}^{\prime 0}=0$, and neglecting the effect of

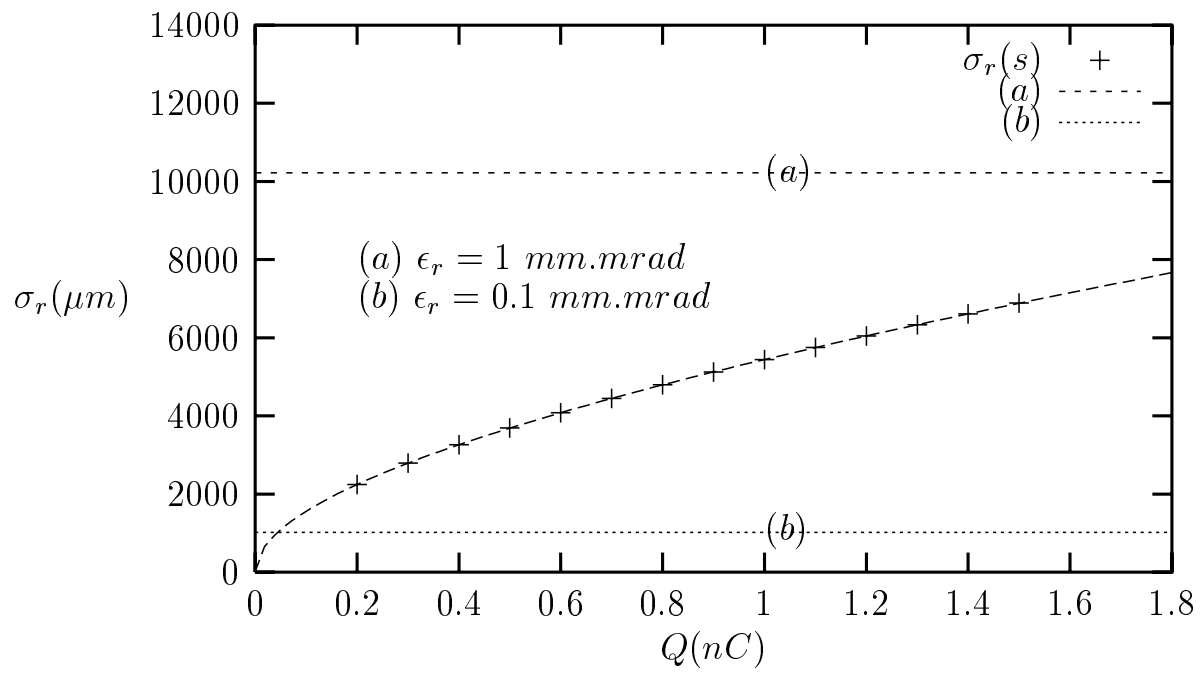

FIGURE 6. Transverse beam size (at $s=50 \mathrm{~mm}$ ) as a function charge $Q$ for a SM-LWFA bunch. The horizontal lines are for no space charge and unnormalized transverse emittances of 0.1 and $1 \mathrm{~mm}$-mrad. 


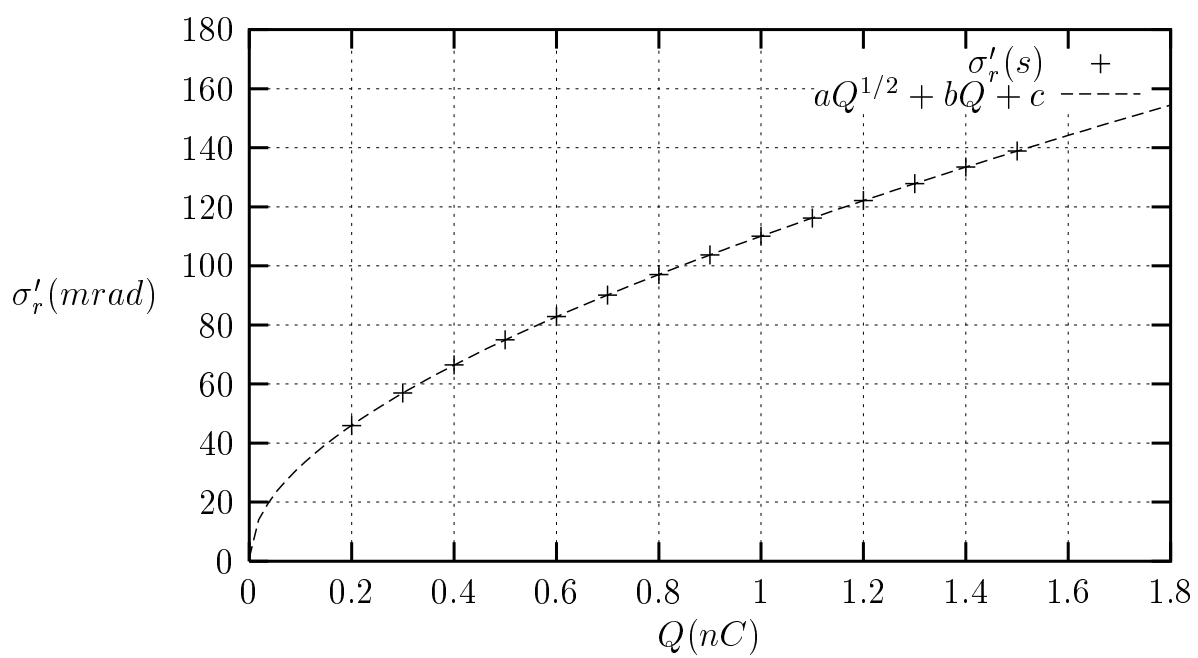

FIGURE 7. Beam divergence (at $s=50 \mathrm{~mm}$ ) as a function of charge $Q$ for a SM-LWFA bunch. Points are from a numerical calculation and the curve represents a fit of the form $a Q^{1 / 2}+b Q+c$, where $a, b$ and $c$ are constants.

emittance. For comparison with the emittance dominated regime, the solution of Eq. (9) is shown for an unnormalized transverse rms emittance $\epsilon_{r}=1.0 \mathrm{~mm}-\mathrm{mrad}$ and $\epsilon_{r}=0.1 \mathrm{~mm}-\mathrm{mrad}$.

One can also study the average beam divergence $\left\langle\sigma_{r}^{\prime 2}\right\rangle^{1 / 2}$ and $\left\langle\sigma_{s}^{\prime 2}\right\rangle^{1 / 2}$, where

$$
\left\langle\sigma_{r}^{\prime 2}\right\rangle=\sum_{i=0}^{n} N_{i} \sigma_{r i}^{\prime 2} / \sum_{i=0}^{n} N_{i}, \quad\left\langle\sigma_{s}^{\prime 2}\right\rangle=\sum_{i=0}^{n} N_{i} \sigma_{s i}^{\prime 2} / \sum_{i=0}^{n} N_{i} .
$$

Figure 7 shows the transverse beam divergence as a function of charge for the parameters of Fig. 6. The divergence $\left\langle\sigma_{r}^{\prime 2}\right\rangle^{1 / 2}$ is clearly dependent of the beam characteristics $\left(\sigma_{r}^{0}, \sigma_{s}^{0}, Q, \gamma_{0}\right)$, but for fixed sizes $\left\langle\sigma_{r}^{\prime}\right\rangle^{1 / 2}$ is in good agreement with a fit of the form $a Q^{1 / 2}+b Q+c$, where $a, b$ and $c$ are constants, which can be used as an empirical scaling law.

\section{CONCLUSION}

Plasma-based accelerators offer the possibility of providing compact, high energy electron accelerators and are also capable of producing ultrashort electron bunches in which the longitudinal size is much smaller than the transverse size. Space charge effects are not of concern while the bunch is in the plasma wave, since the longitudinal and transverse fields of the wake are typically much greater than the space charge forces of the bunch, but space charge cannot be neglected when an electron bunch propagates in vacuum with no external fields, because of its very compact dimensions. The evolution of the bunch sizes under the influence of space charge has been considered with the assumptions that the beam shape 
remains ellipsoidal, and the charge density within the bunch is uniform. Space charge effects on bunches produced in the SM-LWFA and colliding pulse scheme have been examined computationally. Transverse and longitudinal beam growth and the normalized emittance growth depend strongly on energy, but even a fairly high energy electron bunch $\sim 40 \mathrm{MeV}$ can still be space charge dependent if it contains a high charge density.

The above analysis and simulations assumed that within the ellipsoidal bunch, the charge density was uniform. It is possible to perform a similar analysis that assumes the charge density is Gaussian, although this formulation is somewhat more difficult numerically. Previous studies [16] have found that the uniform charge model tends to under estimate the effects of space charge compared to the Gaussian charge distribution model, since a Gaussian distribution gives a higher charge density near the bunch center. Hence, the results presented in this paper present an lower bound on the effects of space charge on ultrashort bunches.

\section{ACKNOWLEDGMENTS}

The authors acknowledge useful discussions with David Bruhwiler and John Staples. This work was supported by the U.S. Department of Energy, Contract No. DE-AC-03-76SF0098.

\section{REFERENCES}

1. For a review see, E. Esarey et al., IEEE Trans. Plasma Sci. PS-24, 252 (1996).

2. E. Esarey et al., Phys. Rev. Lett. 79, 2682 (1997).

3. C.B. Schroeder et al., Phys. Rev. E 59, 6037 (1999).

4. E. Esarey et al., Phys. Plasmas 6, 2262 (1999).

5. J.D. Jackson, Classical Electrodynamics (Wiley, 1975).

6. R.B. Miller, An Introduction to the Physics of Intense Charged Particle Beams (Plenum, 1985).

7. DESY Report, DESY 88-013, 1988.

8. DESY Report, DESY 87-161, 1987.

9. O.D. Kellogg, Foundations of potential theory (Dover, 1953).

10. M. Reiser, Theory and design of charged particle beams (Wiley, 1994).

11. A. Modena et al., Nature 377, 606 (1995); D. Gordon et al., Phys. Rev. Lett. 80, 2133 (1998).

12. K. Nakajima et al., Phys. Rev. Lett. 74, 4428 (1995).

13. D. Umstadter et al., Science 273, 472 (1996); R. Wagner et al., Phys. Rev. Lett. 78, 3125 (1997).

14. A. Ting et al., Phys. Plasmas 4, 1889 (1997); C.I. Moore et al., Phys. Rev. Lett. 79, 3909 (1997).

15. W.P. Leemans, et al., these proceedings.

16. David Bruhwiler, private communication. 